\title{
Für eine Soziologie aus Fleisch und Blut*
}

\author{
Loïc Wacquant
}

\begin{abstract}
Der Artikel diskutiert eine Reihe von Punkten rund um Pierre Bourdieus dispositionelle Handlungstheorie. Dabei geht es zunächst um eine Kritik der problematischen Vorstellungen vom (dualistischen) handelnden Subjekt, wie sie in den gegenwärtigen Sozialwissenschaften vorherrschen. Stattdessen wird ein alternatives Konzept des sozialen Wesens vorgeschlagen: die sinnliche, leidende, kunstfertige, vielschichtige und kontextspezifisch handelnde, körperliche Kreatur. Die Vorrangstellung des verkörperten praktischen Wissens wird betont und es wird diskutiert, mit welchen Forschungsmethoden diese körperorientierte Konzeption des menschlichen Wesens angewandt und gefördert werden kann. Dabei wird argumentiert, dass vor allem die enactive ethnography - eine Form der intensiven Feldforschung, die darauf basiert, ein Phänomen im Forschungsprozess aktiv auszuführen und zu durchleben - dazu geeignet ist, kognitive, konative und emotionale Schemata aufzudecken, die den Praktiken und der zu erforschenden Welt zugrunde liegen.
\end{abstract}

\footnotetext{
* Bei diesem Beitrag handelt es sich um eine leicht gekürzte Übersetzung. Die englische Originalfassung erscheint im Frühjahr 2015 im Sammelband Revealing Tacit Knowledge: Embodiment and Explication im Transcript Verlag (Adloff et al. 2015). Die Übersetzung und deutsche Veröffentlichung des Textes beruht auf einer Kooperation zwischen den beiden Zeitschriften Berliner Debatte Initial und s u b \urban. Die komplette Fassung erscheint zusammen mit einer Reihe von Repliken in Berliner Debatte Initial, Heft 2/2015.
}

Bei dem folgenden Artikel handelt es sich um die erweiterte Fassung eines Textes, der als Antwort auf einige Fragen entstand, die Frank Adloff (Soziologe an der Friedrich-Alexander-Universität Nürnberg und am European University Institute in Florenz) und seine Kolleg_innen an mich richteten, um sie in einen Sammelband zur Beziehung von „Verkörperung und Explikation" in der Sozialanalyse zu integrieren.[1] Die Fragen dienen mir hier als Ausgangspunkt für die Klärung einiger Diskussionspunkte rund um Pierre Bourdieus dispositionelle Handlungstheorie und sind Teil der in den letzten zehn Jahren geführten Debatten, an denen ich im Zuge meiner körperbezogenen [incarnate] Studien zum Profiboxkampf als ,plebejischer Körperertüchtigung beteiligt war.[2]

Im Folgenden werde ich zunächst die problematischen Vorstellungen vom (dualistischen) handelnden Subjekt, von (äußerlicher) Struktur und von (vergeistigtem [mentalist]) Wissen kritisieren, wie sie in den gegenwärtigen Sozialwissenschaften vorherrschen. Stattdessen schlage ich ein alternatives Konzept des sozialen Wesens [social animal] vor. Dieses wird nicht nur als neokantianische Schöpfer_in von und Herrscher_in über Symbole charakterisiert - in der Tradition von Ernst Cassirer, George Herbert Mead und John Searle (2009: ix) werden Menschen als aufmerksame, vernünftige, Sprechakte vollziehende, soziale und politische Wesen mit einem freien Willen gedacht -, sondern als eine sinnliche, leidende, kunstfertige, vielschichtige und kontextspezifisch handelnde, körperliche Kreatur. Dabei 
betone ich die Vorrangstellung des verkörperten praktischen Wissens, das zugleich hervorgeht aus sowie beständig verwoben ist mit Netzen sozialen Handelns, aus denen sich Diskursmacht/-herrschaft entwickelt und auferlegt. Dann diskutiere ich, mit welchen Forschungsmethoden diese körperorientierte Konzeption des menschlichen Wesens angewandt und gefördert werden kann. Ich argumentiere, dass vor allem die handelnde Ethnografie [enactive ethnography] - eine Form der intensiven Feldforschung, die darauf basiert, ein Phänomen [im Forschungsprozess, Anm. d. Ü.] aktiv auszuführen und zu durchleben - dazu geeignet ist, kognitive, konative[3] und emotionale Schemata (also den Habitus) aufzudecken, die den Praktiken und der zu erforschenden Welt zugrunde liegen. Aber es braucht Mut und Ausdauer, um sich eine angemessene Position für die ,teilnehmende Beobachtung' zu erarbeiten und diese analytisch auszuschöpfen.

\section{Wie wichtig ist es Ihrer Meinung nach, auf die impliziten Dimensionen sozialer Interaktionen (implizites Wissen [tacit knowledge], praktisches Wissen, praktischer Sinn [sens pratique] etc.) zu fokussieren?}

Gerade dieser Fokus scheint mir zentral, wenn wir drei notorische Mängel überwinden wollen, welche die Sozialwissenschaften einschränken und uns daran hindern, ein lebendiges und umfassendes Verständnis von der Gesellschaft und ihrer Geschichte zu entwickeln: erstens eine dualistische und leblose [disincarnated] Akteurskonzeption, die darauf beruht, dass dem handelnden Subjekt zwar ein aktiver Verstand zugestanden wird, dieser aber in einem leblosen und untätigen, konzeptionell abwesenden Körper haust; zweitens ein verflachter und negativer Begriff von Struktur, der einfach nur als Set äußerer Zwänge und Beschränkungen daherkommt; und drittens ein vergeistigtes Verständnis von Wissen, das als Konglomerat unterschiedlicher Informationsbrocken und Repräsentationsbestände konzipiert wird. Diese drei verkürzten Begriffe verstärken einander gegenseitig. Zusammen nehmen sie dem sozialen Leben buchstäblich seine Lebendigkeit und lassen uns zurück mit einem unvollständigen und unangemessenen Verständnis des Sozialen als einer fluiden und doch irgendwie strukturierten Handlungssphäre.

Denken wir einmal kurz über jeden dieser Begriffe nach: Quer durch die Sozialwissenschaften finden wir Konzeptionen des handelnden Subjekts entlang von zwei Gegensätzen. Sie bewegen sich zwischen dem homo oeconomicus auf der einen Seite, also der rationalen Rechenmaschine, die den individuellen Nutzen maximiert - diese Vorstellung vom handelnden Subjekt geht auf Bentham zurück und wurde von der neoklassischen Ökonomie weiterentwickelt - und dem homo culturalis auf der anderen Seite, also dem Symbole handhabenden, durch Moral und Normen motivierten Individuum, das auf Kant zurückgeht und von der Kulturanthropologie hochgehalten wird. Unbeholfen erstreckt die Soziologie sich von einem Pol zum anderen. Die beiden herrschenden Modelle - der ,rational man und der ,plastic man', wie Martin Hollis (1977) sie einmal charakterisiert hat - sind zugleich verstümmelt und verstümmelnd. Was ist ihnen trotz ihrer Gegensätzlichkeit und darüber hinaus gemein? Beide ignorieren den 
Körper in dem Sinne, dass sie von Fleisch, Sehnsucht und Leidenschaft als Modalitäten der sozialen Existenz absehen. Genau dies sind die Elemente des Handelns, mit denen William James sich auseinandergesetzt hat und die Sigmund Freuds Tiefenpsychologie erfassen sollte, allerdings nur auf einer ontogenetischen Ebene. Die verschiedenen Stränge der Kognitionswissenschaft in den Forschungsbereichen der künstlichen Intelligenz, der Psychologie, Neurobiologie, Linguistik und Philosophie entdecken dies gegenwärtig auf phylogenetischer Ebene wieder (vgl. Clark 1999, Lakoff/Johnson 1999, Chemero 2013, Shapiro 2014). Aber sie werden zensiert, ignoriert und durch den sozialwissenschaftlichen Mainstream ins Abseits gedrängt.

So ,vielfältig und polymorph' der Begriff nach Merton (1976) auch sein mag, in der vorherrschenden Variante wird ,soziale Struktur' als etwas den handelnden Subjekten äußerliches konzipiert, also als eine Art Zaun oder Trichter. Auch hier haben wir es mit einem Verständnis zu tun, das gleichermaßen verkürzt ist und selbst verkürzend wirkt. Denn Strukturen sind nicht einfach Durkheim'sche Fakten, denen man in der Umwelt, in Form unsichtbarer Beziehungen, in der objektiven Verteilung von Ressourcen oder als Systeme von Beschränkungen und Möglichkeiten begegnet. Vielmehr sind sie auch als dynamische Netze von Kräften tief in den Körper eingeschrieben und wirken dort als Wahrnehmungsraster, als sensomotorische Potenziale und Möglichkeiten, als emotionale Neigungen und tatsächlich auch als Begehren. Strukturen sind ebenso innere Quellen und Antriebskräfte, wie sie äußere Container, Strahlen oder Gitter sind. Sie sind nicht träge und unbeweglich, sondern flexibel und lebendig.

Schließlich arbeiten die Sozialwissenschaften mit einem übermäßig vergeistigten und passiven Wissensbegriff. Wir reservieren den Begriff für aussagenlogische Informationen, die durch Sprache vermittelt und im Verstand verortet werden. Dabei übersehen wir prozedurales und praktisches Wissen, das in den konkreten Taten erworben wird und sich darin auch manifestiert (pragmaticos meint im Altgriechischen: aktiv, geschickt in Bezug auf Geschäfte oder in öffentlichen Angelegenheiten). Mit dem gängigen Top-down-Begriff und dem (ver)geistig(t)en (oder diskursivistischen) Konzept von Kultur, auf dem er basiert, gilt es zu brechen, um - mit Elizabeth Anscombe (1957) gesprochen - die „unverbesserlich kontemplative Konzeption von Wissen " zu überwinden, die uns von der rationalistischen Revolution vererbt wurde. Stattdessen müssen wir die Existenz und Wirkmächtigkeit des in den Körper eingeschriebenen Handlungswissens anerkennen, des intuitiven Verständnisses der sozialen Welt ,von unten “ und zwar sowohl durch intellektuelles Verstehen wie auch durch praktisches Geschick -, das wir durch das Handeln in und unser Einwirken auf unsere soziale Welt erwerben.

\section{Auf welche Eigenschaften des menschlichen Akteurs müssen wir insbesondere achten, wenn wir sein verkörpertes prakti- sches Wissen erfassen wollen?}

Der große neokantianische Philosoph Ernst Cassirer (1944) hat den Menschen zutreffend als , animal symbolicum' charakterisiert. Sprache, 
Mythologie, Kunst, Religion und Wissenschaft waren für ihn die zentralen symbolischen Systeme, die Menschen entwickelt haben, um sich mit ihrer Umwelt auseinanderzusetzen und sie zu formen. Aber diese Eigenschaft allein reicht nicht aus für die Konzeption einer brauchbaren philosophischen Anthropologie. Ich würde sie um fünf weitere ergänzen:

Das animal symbolicum verfügt nicht nur über die Symbole, es ist auch empfindungsfähig, leidensfähig, geschickt [skilled], vielschichtig [sedimented] und komplex sowie räumlich und sozial verortet. Empfindungsfähig: Das handelnde Subjekt ist nicht nur mit außen-, selbst- und introrezeptiven Sinnen ausgestattet; er/sie interpretiert und rationalisiert zudem, was der eigene Wahrnehmungsapparat erfasst. Er/sie ist in der Lage zu fühlen und ist sich der eigenen Gefühle auch bewusst. Und der Körper ist, wie der Neurobiologe Antonio Damasio in The Feeling of What Happens (1999) zeigt, das Medium dieses Gefühlsbewusstseins. Leidensfähig: Das handelnde Subjekt ist Bedrohungen und Rückschlägen durch die natürliche und soziale Welt ausgesetzt; es hat Bedürfnisse, Sehnsüchte und Verlangen, die nicht erfüllt werden; es ist ständig der Beurteilung durch andere unterworfen und mit der Unausweichlichkeit des Todes konfrontiert. So lebt es in Angst, Qual und Schmerz und erträgt seine Existenz doch. Geschickt [skilled]: Das handelnde Subjekt kann ,etwas bewegen' (die ursprüngliche Bedeutung des altnordischen skil ist, verstehen und anpassen'), weil es durch Übung und Erfahrung die Fähigkeit entwickelt hat, zu handeln, und die Fertigkeit, Aufgaben kompetent zu erledigen. Vielschichtig: Alle diese Elemente, unsere Sinne, unsere Leidensfähigkeit und Geschicklichkeit sind uns nicht qua Geburt, also gattungsgemäß, gegeben oder entstehen in der Beschäftigung mit uns selbst. Vielmehr wachsen sie über die Zeit hinweg, werden kultiviert und kommen in der Auseinandersetzung mit der Welt zur Anwendung. Sukzessive werden sie als das Produkt unserer unterschiedlichen, individuellen und kollektiven Geschichten in unsere Körper eingelagert. (Im Anschluss an Husserl bezeichnet Merleau-Ponty [1945/1962] das „habituelle Wissen der Welt“, das im Körper selbst untergebracht ist, als eine „,implizite oder sedimentierte Wissenschaft“.) Verortet: Die sukzessive Einlagerung von Erfahrung wird durch unsere einzigartige Verortung und Bewegung im physischen und sozialen Raum geprägt, gerade weil wir von dem physischen und dabei fragilen Körper unseres sterblichen Organismus zugleich geschützt und eingeschlossen sind. Obwohl er nicht an zwei Orten zur gleichen Zeit sein kann, trägt er doch die Spuren der vielen Orte in sich, die wir über die Zeit hinweg aufgesucht haben.

Schlussendlich werden alle diese sechs Elemente strukturiert, und sie fließen und wachsen im Zeitverlauf. Unsere Vorstellungen vom handelnden Subjekt, von Struktur und Wissen müssen alle radikal zeitlich gedacht werden. Darauf hat Bourdieu bereits in „The work of time“ (1980/1990: 98-111) hingewiesen. Wenn wir erst einmal anerkennen, dass Erkenntnis eine verortete Aktivität ist, die aus einem verworrenen Tanz von Körper, Verstand, Aktivität und der Welt erwächst, können wir beginnen, uns das implizite Wissen [tacit knowledge] aus den Tiefen kultureller und sozialer Praktiken zu erschließen, und dadurch unsere Beschreibungen dieser Praktiken bereichern und ihre Erklärungen ausbauen. Diese drei umgearbeiteten Elemente zusammengenommen - das körperliche Wesen, welches 
praktisches Wissen anwendet, um aktive und mobile Konfigurationen von Affekt, Handlung und Macht zu navigieren -, bilden die Bausteine einer Soziologie aus Fleisch und Blut. Damit sind wir in der Lage, multidimensionale, polychrome Erzählungen über das soziale Leben zu produzieren, die eben dieses Leben erfassen, so wie es sich tatsächlich entfaltet - anstatt leidenschaftslose Schwarz-Weiß-Berichte zu verfassen, wie wir sie derzeit in den akademischen Journals zu lesen bekommen.

\section{Welche Forschungsmethoden bieten sich an, um den unsicht- baren Dimensionen von Handlung, Struktur und Wissen nach- zuspüren?}

Im Prinzip kann man mit den vier Hauptmethoden der Sozialwissenschaften - der Ethnografie, der Hermeneutik (einschließlich der Interview- wie auch der Textanalyse), der historischen und der statistischen Methode - jeden Gegenstand untersuchen. Aber es ist klar, dass sie unterschiedlich gut ausgerüstet sind, um jene Komponenten der Praxis aufzuspüren, die nicht als solche artikuliert, symbolisiert und objektiviert werden: doxische Kategorien, Fertigkeiten, die auf praktischem Wissen basieren, und alltägliche Formen des Lebens, Fühlens und Handelns. Den methodischen Königsweg zum Verständnis der impliziten Beschaffenheit [texture] des sozialen Handelns und Wissens [cognition] bildet die auf direkter Beteiligung an den zu erforschenden Aktivitäten beruhende Nahbeobachtung.

Die Ethnografie - eine spezifische Technik der Datengewinnung und -analyse, die darauf beruht, den sachkundigen und empfindsamen Organismus der Beobachter_in als zentrales Untersuchungswerkzeug einzusetzen - hilft uns wie keine andere Methode, die Gesellschaft zu verkörperlichen, indem die praxeologischen Dimensionen der sozialen Existenz wiederhergestellt und damit nachvollzogen werden. Doch dazu müssen wir erstens ein besseres Verständnis der Besonderheiten und speziellen Eigenschaften (wie auch der entsprechenden Grenzen) der Ethnografie entwickeln. Denn diese ist eine sozial eingebettete und verkörperlichte soziale Untersuchungsmethode, die auf der physischen Kopräsenz der Forschenden - in Echtzeit und -raum - mit und innerhalb des zu erforschenden Phänomens basiert. Zweitens müssen wir unsere ethnografische Praxis in zweierlei Hinsicht reformieren, und zwar in ergänzender und dabei scheinbar widersprüchlicher Art und Weise. Einerseits müssen wir die Ethnografie enger an die sozialwissenschaftliche Theoriebildung anbinden, im Gegensatz zur epistemologischen Illusion der Geertz'schen ,dichten Beschreibung;, der philosophischen Naivität des Empirismus der Chicagoer Schule und der glamourösen Verführungen des postmodernen Geschichten-Erzählens (Wacquant 2002: 1469-71, 1481-82; 2009: 118-122). Andererseits müssen wir gerade die langfristigen, intensiven und initiatorischen Formen der ethnografischen Beteiligung pflegen. Denn nur diese ermöglichen es dem Forscher/der Forscherin, die prädiskursiven Schemata - intus et in cute - zu verstehen, die das Denken und Handeln der involvierten Subjekte in dem zu untersuchenden Universum prägen.

Will die Feldforscher_in die Potenziale der Ethnografie voll ausschöpfen, muss der Fakt anerkannt und methodisch umgesetzt werden, dass er oder sie, wie jedes handelnde Subjekt, den Untersuchungsgegenstand körperlich 
erfährt. Indem man sich sozial und symbolisch tief in das Universum integriert, das man studiert, kann man den Prozess des körperlichen Verständnisses für sich nutzbar machen. Das heißt, wir können und sollten daran arbeiten, in der Praxis unserer Feldforschung zu ,verwundbaren Beobachter_innen ' zu werden - und zwar nicht auf dem Papier, indem wir ,verwundbar schreiben und eine gehörige Portion „Subjektivität in die Ethnografie“ einfließen lassen, wie Ruth Behar es vorschlägt (1996: 16 und 6). Zwar gilt es, möglichst tief in den Fluss des Geschehens einzutauchen, anstatt ihn nur vom Ufer aus zu beobachten. Doch dabei muss die Forscher_in mit Intention und Methode eintauchen und schwimmen. Denn die leichtfertige Hingabe würde dazu führen, dass wir im bodenlosen Strudel der Subjektivität ertrinken.

\section{In Ihrem Buch Body and Soul und in anderen Essays haben Sie versucht, einen Ansatz zu entwickeln, den Sie als „fleisch- liche Soziologie“ [carnal sociology] bezeichnen: Was unter- scheidet Ihren Ansatz von der auf die sinnliche Wahrnehmung bezogenen [sensual] Ethnografie, die durch die Phänomeno- logie inspiriert ist?}

Pointiert ausgedrückt ist die ,carnal sociology“ keine Soziologie des Körpers im Sinne eines soziokulturellen Gegenstands, sondern entspringt dem Körper als Quelle sozialer Intelligenz und soziologischen Scharfsinns. Das beginnt mit der einfachen Tatsache, dass der handelnde Mensch, wie bereits oben dargelegt, ein empfindungsfähiges und leidendes Wesen aus Fleisch und Blut ist. (Fleisch meint hier die sichtbare Oberfläche des lebendigen Körpers, während Blut sich auf den inneren Kreislauf des Lebens bezieht, das in den Tiefen des Körpers pulsiert - siehe auch Leders (1990) Anmerkungen zu Merleau-Ponty. Ich schließe mich hier George Lakoffs und Mark Johnsons (1999: 3-4 und passim) Ausführungen zu der Frage „Wer wir sind“ an. Für sie ist „der Verstand verkörperlicht und das Denken überwiegend unbewusst“, und die Vernunft ist „,vor allem metaphorisch und imaginär“ sowie auch „emotional beteiligt“.) Die fleischliche Soziologie platziert sich nicht über oder neben der Handlung, sondern an ihrem Produktionsort. Sie versucht die Position des Beobachters zu vermeiden und dabei die Handlung im Prozess selbst [action-in-the-making] zu erfassen, nicht die bereits abgeschlossene Handlung. Sie versucht die Schemata aufzuspüren und zu dokumentieren, die die Praxis prägen und gestalten: die kognitiven, konativen und affektiven Bestandteile des Habitus, dessen Schichtungen und Funktionsweisen der empirischen Forschung voll zugänglich sind (Wacquant 2014a; 2014b). Der Ansatz unterscheidet sich insofern von der auf die sinnliche Wahrnehmung bezogenen Ethnografie, als dass diese ein Forschungsfeld der Sinne darstellt, das einer distinguierten Linie von Simmel, Mauss und Lucien Febvre hin zu Elias und der Lebensphilosophie [dt. im Original] von Arnold Gehlen und Helmut Plessner bis zu aktuellen Strömungen der medizinischen und phänomenologischen Anthropologie folgt. Im Gegensatz dazu sind die ,sensorischen Formationen ' in der fleischlichen [carnal] Soziologie weniger der Forschungsgegenstand (so wie bei Howes 2003) als das Mittel der Forschung.

Die fleischliche Soziologie kann auf jeden Gegenstand bezogen werden und sich einer Vielzahl von Methoden bedienen, solange diese das handelnde 
Subjekt als ein körperliches und sozial eingebettetes konzipieren. Dies betrifft zum Beispiel die Forschung zur Alltagsgeschichte [dt. im Original] und zur Mikrogeschichte sowie die noch junge Historiografie der Gefühle. Regelmäßig bewegen sich diese im Bereich der fleischlichen Soziologie, auch wenn sie es nicht unbedingt wissen oder es ihre Absicht ist. So rekonstruiert Carlo Ginzburg (1976) in Il formaggio e i vermi die Lebenswelt des Müllers Menocchio im 16. Jahrhundert, bevor dieser des Ketzertums angeklagt und verbrannt wurde; Alf Lüdtke (1993) spürt den sozialen Wurzeln und Effekten des Eigensinns [dt. im Original] nach, indem er den hartnäckigen ,eigenen Willen' deutscher Arbeiter_innen in der ersten Hälfte des 20. Jahrhunderts untersucht, der deren widerständige Strategien an der Schnittstelle von Akzeptanz und Widerstand gegen die Macht prägte; Alain Corbin (1988) geht den Veränderungen sensorischer und epistemischer Kulturen nach, in deren Zuge die Meeresküste von einem Ort der Angst zu einem attraktiven Ort der Kontemplation wurde, und die eine neue Haltung gegenüber ,der Natur' hervorbrachten - und sie alle betreiben dabei fleischliche historische Soziologie.

Zur Bearbeitung aktueller Fragestellungen bietet sich vor allem die Methode an, die ich als handelnde, im Sinne einer ausführenden und durchlebenden Ethnografie [enactive ethnography] bezeichne. Dabei handelt es sich um eine umfassende Feldforschung, in deren Zuge die Forscher_in das zu untersuchende Phänomen (bzw. einige Elemente davon) selbst durchlebt, Schicht für Schicht dessen unsichtbare Eigenschaften freilegt und die (hier) wirkenden/ wirksamen Mechanismen testet. Den Ausdruck ,handelnd' [enactive] übernehme ich in diesem Zusammenhang von einem Kollegen aus Berkeley, dem Philosophen Alva Noë, der in seinem Buch Action in Perception vorschlägt, dass Wahrnehmung eine „kunstfertige Aktivität durch den Forscher oder die Forscherin als eine ganzheitliche Person" darstellt (2004: 2). Das finde ich eine sehr passende Beschreibung der Arbeit einer Ethnograf_in (Noë selbst bezieht sich auf die einflussreiche Theorie des „embodied mind“ von Francesco Varela, Evan Thompson und Eleanor Rosch [1991]). Das oberste Gebot dieser Art von Forschung ist es, sich in einer alltäglichen und gewöhnlichen Funktion in die Arena des Handelns zu begeben, sie in einer möglichst ähnlichen Art und Weise zu erkunden wie diejenigen Personen, über die man forscht - seien sie nun Boxer_innen, Professor_innen oder Politiker_innen -, um daraus ein tiefes Verständnis ihres Universums zu entwickeln und Material für die analytische Rekonstruktion zu generieren.

\section{Aber wie verhält es sich mit Situationen, in denen man aus praktischen, rechtlichen oder moralischen Gründen nicht Teil eines bestimmten Phänomens werden kann?}

Natürlich ist es nicht immer einfach, Zugang zu einer bestimmten Gruppe oder Situation zu bekommen und dort teilnehmend zu forschen. Ein aktives Mitglied einer bestimmten Gruppe zu werden, ist oft langwierig und erfordert bestimmte Qualitäten oder Zertifizierungen. Vielleicht besitzt du einfach nicht die Kaltblütigkeit, die es braucht, um Polizist_in zu werden, oder die Geschmeidigkeit von Balletttänzer_innen. Du kannst nicht mal eben Richter_in an einem Gericht werden, und hoffentlich wird dir kein Krankenhaus die Erlaubnis erteilen, Hirnoperationen zum Zwecke 
der soziologischen Forschung durchzuführen. Aber du könntest durchaus Requisiteur_in am Ballett werden, ein Praktikum am Gericht machen oder als Pflegekraft in einem Krankenhaus arbeiten. Es gibt immer mehrere Türen, um auf die Bühne zu gelangen, auf der sich die Handlung abspielt um Goffmans (1967) klassischen Aufsatz mit dem Titel „Where the action is“ zu bemühen. Stets ergeben sich dabei unterschiedliche Möglichkeiten, die Aspekte des Phänomens zu erfahren und mit ihnen zu experimentieren und die Rolle des einen oder der anderen Protagonist_in in dem sozialen Drama, um das es geht, zu erlernen und sie selbst einzunehmen - auch wenn es nur eine Nebenrolle sein mag.

Allzu oft stehen Soziolog_innen vor verschlossener Tür, weil sie gar nicht erst anklopfen und fragen, ob ihnen jemand Einlass gewährt. Ein Beispiel: Es gibt Dutzende von Feldforschungen über Obdachlosigkeit, von denen einige ganz vortrefflich gelungen sind - ich empfehle die Lektüre von Snows und Andersons (1993) Down on their Luck, Bourgois' und Schoenbergs (2009) Righteous Dopefiend sowie Gowans (2010) Hobos, Hustlers, and Backsliders. Keine_rdieser Autor_innen hat jedoch längere Zeit auf der Straße, in Billighotels oder Obdachlosenunterkünften geschlafen - ganz zu schweigen von einem beständigen Wechsel zwischen diesen drei Orten, die - zusammen mit dem Gefängnis und dem öffentlichen Krankenhaus - das interinstitutionelle Netz bilden, das mit Wohnungslosen umgeht, wenn ihr Status akut problematisch wird. (Man muss schon bis zu der durch die WPA[4] inspirierten Arbeit von Sutherland und Locke [1936] zurückgehen, um eine Studie zu finden, in der das Übernachten in Obdachlosenunterkünften zu einem Kernelement des Forschungsdesigns gehört.) In der Forschung über Obdachlosigkeit verlässt man das Feld typischerweise am Abend, also ausgerechnet dann, wenn sich die praktische und oftmals existenzielle Frage stellt: Wo werde ich heute Nacht schlafen? Dabei kann man so viel davon lernen, einmal selbst die unterschiedlichen Techniken auszuprobieren, die Obdachlose entwickeln, um sich Nacht für Nacht ein ,Bett‘ auf der Straße zu machen; einmal selbst die fragilen Beziehungen zu regeln, derer es bedarf, um ein Minimum an Sicherheit herzustellen, die Schmerzen zu unterdrücken und die Gefühle im Zaum zu halten, die Veränderung von Geruch und Zeitgefühl auf der Straße zu erleben usw. So viele Facetten des Phänomens können am besten durch den Körper entdeckt werden, im ,Durchleben' [enactment] der Obdachlosigkeit selbst - auch wenn dies im Falle der Forschenden vorsätzlich und temporär sein mag. Denn all diese Elemente sind tief in das Gefüge dringender und dabei doch banaler Handlungen eingelassen und werden von denen, die sich daran gewöhnt haben, teilweise gar nicht mehr wahrgenommen und dementsprechend auch nicht mehr explizit beschrieben.

Diese Art der persönlich und körperlich involvierten Forschung braucht ein bisschen Mut und viel Ausdauer. Die meisten Ethnograf_innen fallen in eine textorientierte und hermeneutische Vorstellung der sozialen Welt zurück, die sich aus einer kontemplativen Haltung speist (per Definition unmöglich für diejenigen, die ,teilnehmende Beobachtung' praktizieren), weil sie die Körperlichkeit von Handlung, Wissen und Struktur übersehen. Darüber hinaus sind viele Forscher_innen einfach nicht ausdauernd genug, wenn es darum geht, sich einen Zugang zum Feld zu erarbeiten und die eigene Einbettung zu vertiefen. Anstatt nach unterschiedlichen 
Zugangsmöglichkeiten zu suchen, zieht man sich schnell zurück. Aber Beharrlichkeit zahlt sich aus: Joan Cassell (1991) zum Beispiel wurde anfänglich der Zugang zu chirurgischen Krankenhausstationen verwehrt. Stattdessen riet man ihr, doch lieber die Lady's Auxiliary[5] zu studieren. Aufgrund ihrer Hartnäckigkeit gelang es ihr am Ende trotzdem, etwa 200 Operationen in einem Dutzend Krankenhäusern, zwei Gesundheitszentren und einer öffentlichen Klinik zu beobachten. Cassell hatte eine 18-monatige empirische Studie geplant; am Ende verbrachte sie 33 Monate im Feld und durfte schließlich sogar das Operationsbesteck vorbereiten. Gretchen Purser (2012) verbrachte fast drei Jahre damit, sich von Leiharbeitsfirmen in den armen Innenstadtbezirken von Oakland und Baltimore Tagesjobs vermitteln zu lassen. Auf dieser Grundlage schrieb sie eine körperorientierte Erzählung über die sozialen Strategien marginalisierter Tagelöhner_innen an der Schnittstelle von dereguliertem Arbeitsmarkt, Hyperghetto und einem wuchernden Gefängnissystem. Man muss sich schon fragen: Wie gelingt es einer zierlichen, vegan lebenden, weißen Frau mit Brille, im alltäglichen Gerangel mit Exhäftlingen um Jobangebote der schlechtesten Art zu bestehen? Aber sie hat durchgehalten und ist am Ende dieses sozialen Experiments mit einer großartigen Erzählung wieder aufgetaucht, die sich der Fleisch-und-Blut-Realität der flexploitation “ im aufgeblähten Unterleib der US-amerikanischen Ökonomie annimmt.

Fleischliche Soziologie basiert auf einem logischen Schluss und auf einem Wagnis. Der logische Schluss ist der folgende: Wenn es stimmt, dass der Körper nicht nur ein soziales Konstrukt ist, sondern auch ein sozial konstruierender Vektor im Gefüge von Wissen, Praxis und Macht, dann trifft dies auch auf den Körper der Soziolog_in in ihrer Funktion als Forscher_in zu. Das Wagnis besteht darin, zwei Jahrtausende der, Verachtung des Körpers zu überwinden, um Nietzsche zu zitieren. Diese hat uns dazu gebracht, den empfindungsfähigen Organismus als Hindernis auf dem Weg zum Wissen zu interpretieren. Nun müssen wir ihn wieder zu einer üppigen Ressource für die sozialwissenschaftliche Forschung machen. Wenn wir unseren Körper methodisch als ein intelligentes Instrument der praktischen Wissensproduktion anwenden, dann beschleunigen wir damit den Erwerb grundlegender sozialer Kompetenz - die operante Fähigkeit, wie einer von vielen zu fühlen, zu denken und zu handeln. Das ist dann auch ein viel besseres Kriterium zur Beurteilung, wann wir unseren Auftrag als Forscher_in erfüllt haben, als die reine Menge der erhobenen Daten.

\section{Ist die ,handelnde Ethnografie' neu?}

Ich bin versucht zu sagen, dass sie im Gegenteil eine alte und ehrwürdige Form von Ethnografie ist, die auf die Anfänge und das goldene Zeitalter der ethnografischen Forschung zurückgeht. Damals wurden das learning by doing und der enge Kontakt zu ,den Einheimischen` betont - übrigens erinnert Aristoteles uns in seinen Schriften zur Poetik daran, dass die Menschen ihr Wissen zuerst durch Mimesis erlangen. Handelnde und durchlebende Ethnografie macht dieses Prinzip einfach explizit und intensiviert dabei zwei Eigenschaften, die jeder Ethnografie eigen sind, die diesen Begriff verdient: 
dass die Forscher_innen selbst körperlich involviert und in die soziale und symbolische Struktur eingebettet sind, die sie untersuchen. Anders ausgedrückt benutzt sie ,Habitus' sowohl als Gegenstand als auch als Methode der Analyse (vgl. Wacquant 2011): Die Forschenden decken praktische Schemata auf und testen deren Relevanz, a) indem sie sie sich in actu aneignen und intensiv auf die pädagogischen Techniken fokussieren, durch die sie geformt werden; oder b) indem sie die pragmatische Konstruktion analytisch aufschlüsseln, in deren Zuge die Schemata umgesetzt und ausgeführt werden. In dieser Hinsicht wendet sich handelnde Ethnografie gegen jene Strömungen der aktuellen Anthropologie, die von Tropen, Positionalität, Moral, der Offenheit und Vielfalt verschiedener Lagen und Orte [sites] (oder ,parasites“, die „in die Komplexitäten unserer Zeit eingekeilt sind“ - kein Scherz) geradezu besessen sind, und gegen die Eigenheiten der Professionalisierung, die mit ihren Metaanalysen zu einem Zustand der ethnografischen Lähmung geführt haben (vgl. Faubion/Marcus 2009).

Ich habe an anderer Stelle vorgeschlagen, dass wir, immer wenn die praktischen Umstände es zulassen, „uns zu Einheimischen machen können und sollten, aber gut gerüstet, um anschließend auch wieder aus dem Feld zurückzukehren“ (Wacquant 2009: 119). Dieses Gebot ist ein ganz bewusstes Korrektiv zu der im Mainstream der Sozialwissenschaften vorherrschenden Vorstellung von Feldforschung. Ich würde die oben genannte Formulierung gern um einen wichtigen Punkt ergänzen: Das ,uns zu Einheimischen Machen', das ,going native , ist ein missverständliches Motto, das uns auf die falsche epistemologische Fährte führt. Denn was ist die ,einheimische Perspektive', die Malinowski (1922/2014) in The Argonauts of the Western Pacific kanonisierte, und die Geertz (1974) als den Standpunkt der Ethnografie würdigte? Wessen Perspektive ist das und zu welchem Zeitpunkt wird sie eingenommen? Selbst in kleinen und auf Verwandtschaft basierenden Gesellschaften, die sich Durkheims Vorstellung von ,mechanischer Solidarität‘ annähern - wie etwa auf den Trobriand-Inseln -, gibt es soziale Differenzierung und Hierarchien. Und in der Tat betont Malinowski die Unterschiede zwischen Bezirken, Stämmen und totemistischen Gruppen im Hinblick auf Hierarchien und Macht. Offensichtlich ist der Standpunkt eines Dorfoberhauptes nicht der eines gewöhnlichen Gemeindemitglieds, die Perspektive und Einstellung eines älteren männlichen Witwers aus einer hoch angesehenen Familie nicht die eines unverheirateten Mädchens aus einem niedriger gestellten totemistischen Clan. In jedem Handlungssystem gibt es eine Arbeitsteilung, die zu Standpunkten - im Plural - führt, im Sinne unterschiedlicher Perspektiven, die von sich entwickelnden Punkten in der objektiven Struktur des lokalen sozialen Raums aus eingenommen werden. Darüber hinaus ist stets umstritten, welche Eigenschaften und Positionen etwas als ,einheimisch' qualifizieren. Wer gilt als einheimisch und wer nicht? Diese Frage steht immer in der sozialen Welt selbst zur Diskussion und daher sollte sie von den Forschenden auch nicht einfach so auf dem Papier beantwortet werden. Wie ich in The Prizefighter's Three Bodies zeige, basieren die einzelnen Perspektiven, also zum Beispiel die des Boxers oder der Boxerin, nicht einfach auf einer von der ethnografischen Beobachtung ausgehenden, rohen empirischen Induktion. Vielmehr haben wir es immer mit einer selektiven und künstlichen Konstruktion durch den Analytiker oder 
die Analytikerin zu tun, die einen bestimmten Moment im dynamischen und konfliktiven Verhältnis spezifischer Perspektiven zueinander erfasst (vgl. Wacquant im Erscheinen).

Übersetzung aus dem Englischen von Henrik Lebuhn und Johanna Wischner, unter Mitarbeit von Johanna Hoerning, Kristine Müller und Nina Schuster.

\section{Endnoten}

[1] Der Autor dankt Frank Adloff, Javier Auyero, Sarah Brothers, Megan Comfort, Magnus Hörnqvist und Nazli Ökten für ihre Kommentare, die bei der Ausarbeitung dieses Essays sehr geholfen haben.

[2] Siehe vor allem die Sonderausgabe von Qualitative Sociology zum Thema „Body and Soul“ (Sommer 2005), das Symposium zu „Habitus in Body \& Soul“, in Theory \& Psychology (Dezember 2009), und zu „Homines in extremis“ in Body \& Society (Frühling 2014) sowie Wacquant 2009.

[3] Anmerkung des Übersetzers: konativ bedeutet ,auf Absichten bezogen‘.

[4] WPA steht für ,Works Progress Administration‘. 1935 gegründet, war sie die größte US-Bundesbehörde der New-Deal-Ära und entwickelte Arbeitsbeschaffungsprogramme für Millionen von Erwerbslosen (Anm. d. Ü.).

[5] Die Lady's Auxiliary ist eine US-amerikanische Wohltätigkeitsorganisation, die von Frauen getragen wird und sich vor allem der Unterstützung von Kriegsveteran_innen widmet (Anm. d. Ü.).

\section{Autor innen}

Loïc Wacquant ist Soziologe mit Interesse an urbaner Marginalität, Bestrafung, Sinnlichkeit und Sozialtheorie.

loic@berkeley.edu

\section{Literatur}

Adloff, Frank / Gerund, Katharina / Kaldewey, David (Hg.) (2015): Revealing Tacit Knowledge. Embodiment and Explication. Bielefeld: Transcript.

Anscombe, G. E. M. (2000 [1957]): Intention. Cambridge: Harvard University Press.

Auyero, Javier (Hg.) (2005): Editor's note. In: In: Qualitative Sociology, Special Issue on Body and Soul, 28/2, 141-211.

Behar, Ruth (1996): The Vulnerable Observer. Anthropology that Breaks your Heart. Boston: Beacon Press.

Bourdieu, Pierre (1990 [1980]): Le Sens pratique. Paris: Éditions de Minuit. (Englisch: The Logic of Practice. Cambridge: Polity Press, 1990. Deutsch: Sozialer Sinn. Kritik der theoretischen Vernunft. Frankfurt a. M.: Suhrkamp, 1987.)

Bourgois, Philippe / Schonberg, Jeff (2009): Righteous Dopefiend. Berkeley: University of California Press.

Cassell, Joan (1991): Expected Miracles. Surgeons at Work. Philadelphia: Temple University Press.

Cassirer, Ernst (1944): An Essay on Man. New Haven: Yale University Press.

Chemero, Anthony (2013): Radical embodied cognitive science. In: Review of General Psychology 17/2, 145-150. 
Clark, Andy (1999): An embodied cognitive science? In: Trends in Cognitive Sciences 3/9, 345-351.

Corbin, Alain (1988): Le Territoire du vide. L'Occident et le désir du rivage, 1750-1840. Paris: Aubier.

Damasio, Antonio R. (1999): The Feeling of what Happens. Body and Emotion in the Making of Consciousness. New York: Harcourt Brace.

Faubion, James D. / Marcus, George E. (Hg.) (2009): Fieldwork is Not What it Used to Be. Learning Anthropology's Method in a Time of Transition. Ithaca: Cornell University Press.

Geertz, Clifford (1974): 'From the native's point of view'. On the nature of anthropological understanding. In: Bulletin of the American Academy of Arts \& Sciences 28/1, 26-45. (Nachdruck in: ders. (1983): Local Knowledge. Further Essays in the Interpretation of Cultures. New York: Basic Books, 55-71).

Ginzburg, Carlo (1976): Il formaggio e i vermi. Torino: Einaudi.

Goffman, Erving (1967): Interaction Ritual. Essays on Face-to-Face Behavior. New York: Anchor. (Deutsch: Interaktionsrituale. Über Verhalten in direkter Kommunikation. Frankfurt a. M.: Suhrkamp, 1994.)

Gowan, Teresa (2010): Hobos, Hustlers, and Backsliders. Homeless in San Francisco. Minneapolis: University of Minnesota Press.

Hollis, Martin (1977): Models of Man. Philosophical Thoughts on Social Action. New York: Cambridge University Press.

Howes, David (2003): Sensual Relations. Engaging the Senses in Culture and Social Theory. Ann Arbor: University of Michigan Press.

Lakoff, George / Johnson, Mark (1999): Philosophy in the Flesh. The Embodied Mind and its Challenge to Western Thought. New York: Basic Books.

Leder, Drew (1990): Flesh and blood. A proposed supplement to Merleau-Ponty. In: Human Studies 13/3, 209-19.

Lüdtke, Alf (1993): Eigen-Sinn. Fabrikalltag, Arbeitererfahrungen und Politik vom Kaiserreich bis in den Faschismus. Hamburg: Ergebnisse Verlag.

Malinowski, Bronislaw (2014 [1922]): Argonauts of the Western Pacific. An Account of Native Enterprise and Adventure in the Archipelagoes of Melanesian New Guinea. London: Routledge.

Merleau-Ponty, Maurice (1962 [1945]): Phénoménologie de la perception. Paris: Gallimard. (Deutsch: Phänomenologie der Wahrnehmung. Berlin: de Gruyter, 1966/1974).

Merton, Robert (1976): Structural analysis in sociology. In: Peter Blau (Hg.): Approaches to the Study of Social Structure. London: Open Books, 21-52.

Noë, Alva (2004): Action in Perception. Cambridge: MIT Press.

Purser, Gretchen (2012): 'Still doin' time'. Clamoring for work in the day labor industry. In: WorkingUSA: The Journal of Labor \& Society 15/3, 397-415.

Searle, John (2009): Making the Social World. The Structure of Human Civilization. New York: Oxford University Press.

Shapiro, Lawrence (Hg.) (2014): The Routledge Handbook of Embodied Cognition. London: Routledge.

Snow, David A. / Anderson, Leon (1993): Down on their Luck. A Study of Homeless Street People. Berkeley: University of California Press.

Stam, Henderikus J. (Hg.) (2009): Special section on habitus in the work of Loïc Wacquant. In: Theory \& Psychology 19/6, 707-777.

Sutherland, Edwin / Locke, Harvey J. (1936): Twenty Thousand Homeless Men. A Study of Unemployed Men in the Chicago Shelter. Chicago: Lippincott (Nachdruck: New York, Arno Press, 1971).

Varela, Francesco / Thompson, Evan / Rosch, Eleanor (1991): The Embodied Mind. Cognitive Science and Human Experience. Cambridge: MIT Press.

Wacquant, Loï (2004 [2000]): Body and Soul. Notebooks of an Apprentice Boxer. New York: Oxford University Press. Neue erweiterte Auflage 2014. (Deutsch: Leben für den Ring. Boxen im amerikanischen Ghetto. Konstanz: UVK, 2003).

Wacquant, Loïc (2002): Scrutinizing the street. Poverty, morality, and the pitfalls of urban ethnography. In: American Journal of Sociology 107/6, 1468-1532.

Wacquant, Loïc (2009): The body, the ghetto and the penal state. In: Qualitative Sociology 32/1, 101-129. 
Wacquant, Loïc (2011): Habitus as topic and tool. Reflections on becoming a prizefighter. In: Qualitative Research in Psychology 8/1, 81-92. (Deutsch: Habitus als Thema und Analysewerkzeug. Betrachtungen zum Werdegang eines Berufsboxers. In: LiThes. Zeitschrift für Literatur- und Theatersoziologie 4/2010, 5-23.)

Wacquant, Loï (2014a): Homines in extremis. What fighting scholars teach us about habitus. In: Body \& Society 20/2, 3-17.

Wacquant, Loïc (2014b): Putting habitus in its place. A response to the symposium. In: Body \& Society 20/2, 118-139.

Wacquant, Loï (im Erscheinen): The Prizefighter's Three Bodies. Steps to a Carnal Sociology. New York: Oxford University Press. 
